\title{
Specific heat capacities and thermal properties of a homogeneous ethylene-1-butene copolymer by adiabatic calorimetry
}

\author{
P.J. van Ekeren ${ }^{\mathrm{a}, *}$, L.D. Ionescu ${ }^{\mathrm{a}}$, V.B.F. Mathot ${ }^{\mathrm{b}}$, J.C. van Miltenburg ${ }^{\mathrm{a}}$ \\ ${ }^{a}$ Chemical Thermodynamics Group, Debye Institute, Utrecht University, Padualaan 8, 3584 CH Utrecht, The Netherlands \\ ${ }^{\mathrm{b}}$ DSM Research, P.O. Box 18, 6160 MD Geleen, The Netherlands
}

Received 21 September 2001; accepted 23 October 2001

\begin{abstract}
Specific heat capacities of a homogeneous ethylene-1-butene copolymer were measured by adiabatic calorimetry in the temperature range from 7 to $406 \mathrm{~K}$ (stepwise heating after cooling at rates in the range from 6 to $25 \mathrm{~K} \mathrm{~h}^{-1}$, averaged heating rates $5-34 \mathrm{~K} \mathrm{~h}^{-1}$ ). The glass transition is centred around $224 \mathrm{~K}$. With devitrification also melting sets in. The crystallinity of the polymer was calculated (within the two-phase model) as a function of temperature using two sets of reference data (one for linear and branched polyethylenes (BPEs), and the other for strictly linear polyethylene (LPE)) for completely crystalline and for completely amorphous material. On heating, the mass fraction crystallinity decreased from 0.30 to 0 in the temperature range from 220 to $360 \mathrm{~K}$, confirming earlier differential scanning calorimetry (DSC) heat capacity measurements. During the stabilisation periods in the melting region negative temperature drifts, related to endothermic effects caused by melting, were observed below $325 \mathrm{~K}$. However, in the temperature range from $325 \mathrm{~K}$ up to the end melting temperature, $360 \mathrm{~K}$, positive drifts were measured, reflecting exothermic effects which are attributed to recrystallisation phenomena.
\end{abstract}

(C) 2002 Elsevier Science B.V. All rights reserved.

Keywords: Adiabatic calorimetry; Crystallinity; Ethylene-1-butene copolymer; Heat capacity; Polyethylene; Recrystallisation; Relaxation

\section{Introduction}

To support studies on the thermal behaviour of polymers, which are usually performed using differential scanning calorimetry (DSC) [1], it was decided to measure the heat capacity of some ethylene-1-alkene copolymers very accurately over a wide temperature range using adiabatic calorimetry. The results for a heterogeneous ethylene-1-octene very low density

\footnotetext{
* Corresponding author. Tel.: +31-30-2533509; fax: +31-30-2533947.

E-mail address: ekeren@chem.uu.nl (P.J. van Ekeren).
}

polyethylene (VLDPE) with a comonomer content of $6.2 \mathrm{~mol} \%$ and a density of $902 \mathrm{~kg} \mathrm{~m}^{-3}$ and for a homogeneous ethylene-1-octene copolymer with a comonomer content of $13.6 \mathrm{~mol} \%$ and a density of $870 \mathrm{~kg} \mathrm{~m}^{-3}$ have been published previously [2,3]. In this paper, data are presented for a homogeneous ethylene-1-butene copolymer with a comonomer content of $12.9 \mathrm{~mol} \%$ and a density of $878 \mathrm{~kg} \mathrm{~m}^{-3}$. With this contribution we would like to express our appreciation to Dr. M. Richardson and to Dr. G. Höhne for their pioneering research in developing techniques and methods for calorimetry and for their commitment to perform quantitative calorimetry for better understanding of the thermal behaviour of polymers. 


\section{Experimental}

\subsection{The sample}

The sample, EB 5, is a homogeneous ethylene-1butene copolymer $[4,5]$ which was produced using metallocene based catalysis. The molar percentage of 1 -butene is $12.9 \%$ (i.e. the mass percentage of 1-butene is $22.9 \%$ by NMR). Its meltindex, $I_{2}$, is $9.76 \mathrm{dg} / \mathrm{min}$ and the density of the material (at $298 \mathrm{~K}$, after compression moulding) is $878 \mathrm{~kg} \mathrm{~m}^{-3}$.

\subsection{The adiabatic calorimeter}

The measurements were performed using one of our home-built adiabatic calorimeters [6,7]. The temperature is measured using a $27 \Omega$ rhodium-iron resistance, which has been calibrated by Oxford Instruments at 33 points between 1.5 and $300 \mathrm{~K}$. The calibration was extended to $430 \mathrm{~K}$ using the melting temperatures of naphthalene and indium. Conversion to the ITS-90 scale [8] is based on the article of Goldberg and Weir [9].

After filling and successive evacuation of the calorimeter vessel, helium gas is admitted to the vessel until the pressure is about $1000 \mathrm{~Pa}$. Measurements are made in the intermittent mode. This implies that stabilisation periods are followed by input periods under automatic control. During the stabilisation periods, the temperature is recorded as a function of time. Between two stabilisation periods, an input period is used to raise the temperature of the sample (and vessel). The amount of heat added to the calorimeter vessel is measured very accurately. The temperature increase, which is caused by the heat input, follows from extrapolation of the temperature-time curves of both stabilisation periods. These data allow for the accurate calculation of the heat capacity. The heat capacity of the sample is obtained by subtracting the heat capacity of the empty calorimeter vessel. In the transition regions thermal equilibrium is not reached within a practical time limit because of various kinds of temperature-time dependent processes. Therefore, in these regions another method was applied. The known heat transfer to the surroundings (from an empty vessel experiment) and the amount of heat added to the calorimeter vessel were used to calculate the enthalpy increment between (the midpoints of) two successive stabilisation periods. This results in the actual enthalpy path of the vessel and its content.

Due to the adiabatic construction the heat exchange with the surroundings is very small. Therefore, temperature drifts observed in the stabilisation periods may be used to investigate the temperature-time dependent processes. According to measurements of standard materials, the inaccuracy is approximately $0.2 \%$ of the absolute heat capacity.

\subsection{The measurements}

The calorimeter vessel was filled with an amount of $5.52958 \mathrm{~g}$ of the polymer. Eight series of measurements were performed with this sample in the temperature range from 7 to $406 \mathrm{~K}$. An overview of the performed measurements (temperature ranges, duration of stabilisation and input periods and averaged heating rates) is given in Table 1. The sample was cooled after series 1 and 3 by switching off the temperature control of the shields. This resulted in

Table 1

Overview of the series of measurements performed on the EB 5 sample

\begin{tabular}{lcclcc}
\hline Series no. & $T_{1}(\mathrm{~K})$ & $T_{2}(\mathrm{~K})$ & $t$ (stabilisation) $(\mathrm{s})$ & $t$ (input) $(\mathrm{s})$ & $\langle\beta\rangle\left(\mathrm{K} \mathrm{h}^{-1}\right)$ \\
\hline 1 & 298 & 389 & 600 & 708 & 7 \\
2 & 85 & 113 & 600 & 708 & 7 \\
3 & 116 & 385 & 600 & 108 & 3 \\
4 & 7 & 31 & 200 & 100 & 20 \\
5 & 7 & 29 & 600 & 600 & 8 \\
6 & 9 & 100 & 800 & 808 & 5 \\
7 & 98 & 279 & 800 & 808 & 4 \\
8 & 280 & 406 & & & 5 \\
\hline
\end{tabular}

For each measurement series the temperature range in which measurements were performed is given together with the duration of the stabilisation and input periods and the averaged heating rates $\langle\beta\rangle$. 


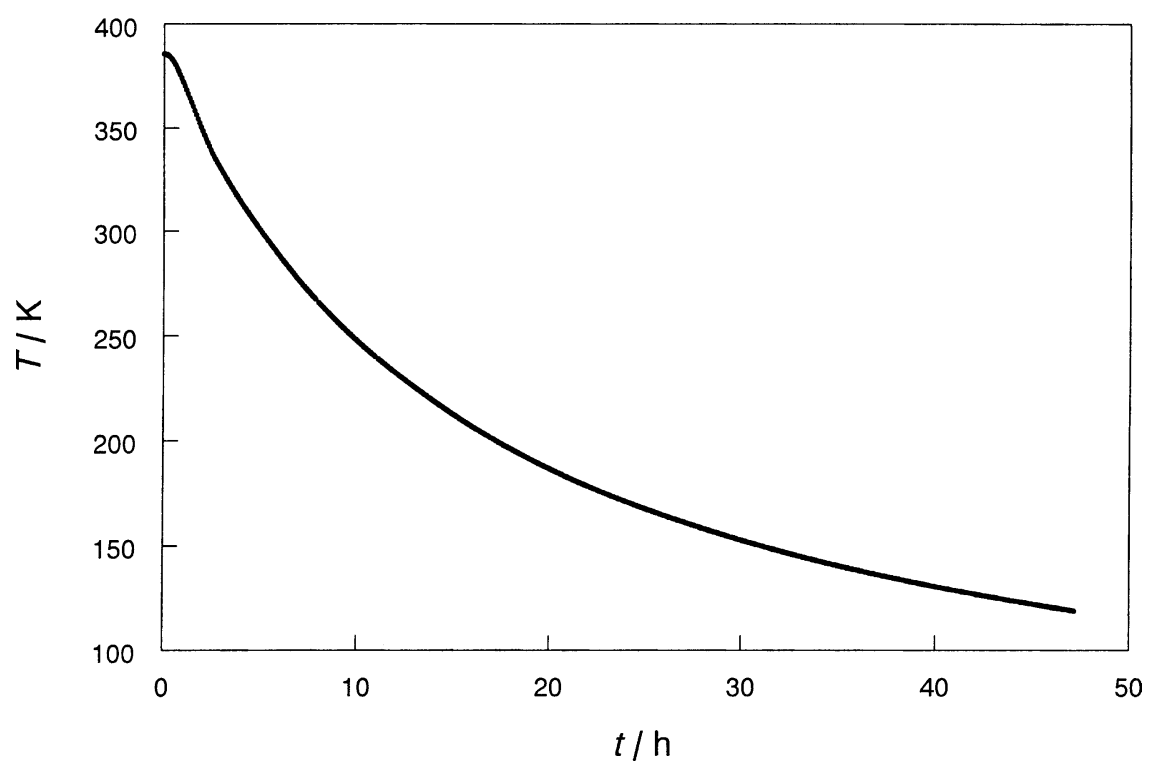

Fig. 1. Cooling curve, recorded after measurement series 3 .

a (slow) exponential decrease of the temperature: at $360 \mathrm{~K}$ the cooling rate was approximately $25 \mathrm{~K} \mathrm{~h}^{-1}$ and at $220 \mathrm{~K}$ the cooling rate was approximately $6 \mathrm{~K} \mathrm{~h}^{-1}$. To have an impression of the thermal history of the sample the cooling curve which was recorded after series 3 is plotted in Fig. 1.

\section{Results and discussion}

\subsection{Specific heat capacity}

The specific heat capacities that were evaluated from the measurements are given in Table 2 and graphically presented in Fig. 2. For temperatures below $315 \mathrm{~K}$ the heat capacities measured in series 1 are considerably lower than those measured in series 3 and 8 at the same temperatures. In the temperature range from 315 to $370 \mathrm{~K}$, on the other hand, the heat capacities measured during series 1 are significantly higher than those measured during series 3 and 8 . This is caused by the different thermal history of the sample for these measurements. Series 1 was performed on the polymer as received, i.e. on material that had been stored for a long time (years) at room temperature. During the storage the material had obviously relaxed to a more stable state. The first measurements of series
Table 2

Measured specific heat capacity of EB 5 as a function of temperature

\begin{tabular}{ll}
\hline$T / K$ & \multicolumn{1}{c}{$C_{p}$} \\
\cline { 2 - 2 } Series 1 & $J K^{-1} g^{-1}$ \\
298.02 & \\
298.96 & 2.384 \\
300.41 & 2.417 \\
302.35 & 2.442 \\
304.29 & 2.480 \\
306.20 & 2.519 \\
308.09 & 2.562 \\
309.95 & 2.613 \\
311.76 & 2.674 \\
313.48 & 2.756 \\
315.08 & 2.882 \\
316.62 & 3.023 \\
318.16 & 3.119 \\
319.71 & 3.170 \\
321.29 & 3.198 \\
322.90 & 3.221 \\
324.52 & 3.244 \\
326.16 & 3.270 \\
327.80 & 3.298 \\
329.44 & 3.330 \\
331.08 & 3.358 \\
332.72 & 3.384 \\
334.34 & 3.403 \\
335.97 & 3.416 \\
337.60 & 3.422 \\
& 3.419
\end{tabular}


Table 2 (Continued)

\begin{tabular}{|c|c|}
\hline$T / K$ & $\frac{C_{p}}{J K^{-1} g^{-1}}$ \\
\hline 339.23 & 3.407 \\
\hline 340.87 & 3.387 \\
\hline 342.52 & 3.351 \\
\hline 344.19 & 3.298 \\
\hline 345.88 & 3.222 \\
\hline 347.62 & 3.114 \\
\hline 349.42 & 2.979 \\
\hline 351.27 & 2.831 \\
\hline 353.19 & 2.693 \\
\hline 355.15 & 2.580 \\
\hline 357.16 & 2.507 \\
\hline 359.18 & 2.462 \\
\hline 361.22 & 2.439 \\
\hline 363.24 & 2.428 \\
\hline 365.25 & 2.423 \\
\hline 367.26 & 2.424 \\
\hline 369.25 & 2.428 \\
\hline 371.25 & 2.433 \\
\hline 373.24 & 2.439 \\
\hline 375.23 & 2.445 \\
\hline 377.22 & 2.452 \\
\hline 379.21 & 2.454 \\
\hline 381.20 & 2.452 \\
\hline 383.20 & 2.460 \\
\hline 385.21 & 2.464 \\
\hline 387.21 & 2.467 \\
\hline 389.21 & 2.472 \\
\hline \multicolumn{2}{|l|}{ Series 2} \\
\hline 85.31 & 0.605 \\
\hline 87.75 & 0.621 \\
\hline 90.39 & 0.637 \\
\hline 93.25 & 0.654 \\
\hline 96.11 & 0.670 \\
\hline 98.97 & 0.686 \\
\hline 101.83 & 0.706 \\
\hline 104.71 & 0.720 \\
\hline 107.59 & 0.734 \\
\hline 110.48 & 0.751 \\
\hline 113.44 & 0.683 \\
\hline \multicolumn{2}{|l|}{ Series 3} \\
\hline 115.78 & 0.777 \\
\hline 117.70 & 0.788 \\
\hline 120.13 & 0.801 \\
\hline 123.05 & 0.817 \\
\hline 125.97 & 0.833 \\
\hline 128.89 & 0.848 \\
\hline 131.81 & 0.863 \\
\hline 134.73 & 0.878 \\
\hline 137.66 & 0.893 \\
\hline 140.58 & 0.910 \\
\hline 143.50 & 0.925 \\
\hline 146.42 & 0.940 \\
\hline 149.35 & 0.955 \\
\hline
\end{tabular}

Table 2 (Continued)

\begin{tabular}{|c|c|}
\hline$T / K$ & $\frac{C_{p}}{J K^{-1} g^{-1}}$ \\
\hline 152.28 & 0.971 \\
\hline 155.21 & 0.987 \\
\hline 158.14 & 1.002 \\
\hline 161.07 & 1.020 \\
\hline 163.99 & 1.036 \\
\hline 166.91 & 1.052 \\
\hline 169.83 & 1.069 \\
\hline 172.76 & 1.087 \\
\hline 175.68 & 1.104 \\
\hline 178.60 & 1.122 \\
\hline 181.52 & 1.139 \\
\hline 184.44 & 1.159 \\
\hline 187.36 & 1.176 \\
\hline 190.29 & 1.195 \\
\hline 193.21 & 1.213 \\
\hline 196.13 & 1.232 \\
\hline 199.04 & 1.252 \\
\hline 201.95 & 1.274 \\
\hline 204.87 & 1.294 \\
\hline 207.76 & 1.329 \\
\hline 210.65 & 1.370 \\
\hline 213.50 & 1.411 \\
\hline 216.29 & 1.459 \\
\hline 219.02 & 1.517 \\
\hline 221.69 & 1.586 \\
\hline 224.30 & 1.653 \\
\hline 226.85 & 1.709 \\
\hline 229.36 & 1.762 \\
\hline 231.84 & 1.805 \\
\hline 234.27 & 1.850 \\
\hline 236.68 & 1.890 \\
\hline 239.06 & 1.927 \\
\hline 241.41 & 1.964 \\
\hline 243.73 & 2.000 \\
\hline 246.03 & 2.034 \\
\hline 248.32 & 2.067 \\
\hline 250.57 & 2.100 \\
\hline 252.81 & 2.132 \\
\hline 255.03 & 2.156 \\
\hline 257.23 & 2.186 \\
\hline 259.41 & 2.216 \\
\hline 261.58 & 2.247 \\
\hline 263.72 & 2.278 \\
\hline 265.85 & 2.307 \\
\hline 267.95 & 2.338 \\
\hline 270.04 & 2.369 \\
\hline 272.11 & 2.397 \\
\hline 274.16 & 2.427 \\
\hline 276.20 & 2.459 \\
\hline 278.22 & 2.490 \\
\hline 280.22 & 2.519 \\
\hline 282.21 & 2.550 \\
\hline 284.19 & 2.575 \\
\hline
\end{tabular}


Table 2 (Continued)

\begin{tabular}{|c|c|}
\hline$T / K$ & $\frac{C_{p}}{J K^{-1} g^{-1}}$ \\
\hline 286.14 & 2.610 \\
\hline 288.08 & 2.640 \\
\hline 290.01 & 2.669 \\
\hline 291.92 & 2.699 \\
\hline 293.82 & 2.726 \\
\hline 295.70 & 2.754 \\
\hline 297.58 & 2.783 \\
\hline 299.44 & 2.808 \\
\hline 301.29 & 2.832 \\
\hline 303.14 & 2.860 \\
\hline 304.97 & 2.884 \\
\hline 306.80 & 2.910 \\
\hline 308.61 & 2.936 \\
\hline 310.42 & 2.963 \\
\hline 312.22 & 2.990 \\
\hline 314.00 & 3.019 \\
\hline 315.78 & 3.048 \\
\hline 317.55 & 3.080 \\
\hline 319.30 & 3.114 \\
\hline 321.04 & 3.149 \\
\hline 322.77 & 3.182 \\
\hline 324.48 & 3.217 \\
\hline 326.19 & 3.246 \\
\hline 327.89 & 3.271 \\
\hline 329.59 & 3.290 \\
\hline 331.28 & 3.302 \\
\hline 332.98 & 3.309 \\
\hline 334.68 & 3.311 \\
\hline 336.38 & 3.312 \\
\hline 338.09 & 3.311 \\
\hline 339.80 & 3.304 \\
\hline 341.51 & 3.284 \\
\hline 343.24 & 3.246 \\
\hline 344.98 & 3.186 \\
\hline 346.74 & 3.098 \\
\hline 348.55 & 2.982 \\
\hline 350.40 & 2.842 \\
\hline 352.30 & 2.687 \\
\hline 354.26 & 2.545 \\
\hline 356.28 & 2.449 \\
\hline 358.32 & 2.408 \\
\hline 360.37 & 2.398 \\
\hline 362.43 & 2.398 \\
\hline 364.48 & 2.401 \\
\hline 366.53 & 2.407 \\
\hline 368.58 & 2.415 \\
\hline 370.62 & 2.422 \\
\hline 372.65 & 2.430 \\
\hline 374.69 & 2.438 \\
\hline 376.72 & 2.444 \\
\hline 378.74 & 2.448 \\
\hline 380.77 & 2.452 \\
\hline 382.79 & 2.454 \\
\hline
\end{tabular}

Table 2 (Continued)

\begin{tabular}{|c|c|}
\hline$T / K$ & $\frac{C_{p}}{J K^{-1} g^{-1}}$ \\
\hline 384.81 & 2.458 \\
\hline \multicolumn{2}{|l|}{ Series 4} \\
\hline 6.61 & 0.009 \\
\hline 7.26 & 0.012 \\
\hline 8.59 & 0.017 \\
\hline 10.45 & 0.027 \\
\hline 12.16 & 0.036 \\
\hline 14.07 & 0.049 \\
\hline 16.17 & 0.063 \\
\hline 18.37 & 0.079 \\
\hline 20.67 & 0.097 \\
\hline 23.06 & 0.117 \\
\hline 25.53 & 0.137 \\
\hline 28.09 & 0.154 \\
\hline 30.54 & 0.171 \\
\hline \multicolumn{2}{|l|}{ Series 5} \\
\hline 7.14 & 0.011 \\
\hline 8.28 & 0.016 \\
\hline 9.73 & 0.023 \\
\hline 11.49 & 0.032 \\
\hline 13.19 & 0.044 \\
\hline 14.94 & 0.054 \\
\hline 16.80 & 0.068 \\
\hline 18.71 & 0.083 \\
\hline 20.66 & 0.099 \\
\hline 22.66 & 0.115 \\
\hline 24.70 & 0.134 \\
\hline 26.78 & 0.152 \\
\hline 28.94 & 0.168 \\
\hline \multicolumn{2}{|l|}{ Series 6} \\
\hline 9.43 & 0.023 \\
\hline 13.23 & 0.042 \\
\hline 15.61 & 0.057 \\
\hline 17.61 & 0.073 \\
\hline 19.72 & 0.091 \\
\hline 21.91 & 0.108 \\
\hline 24.07 & 0.128 \\
\hline 26.22 & 0.147 \\
\hline 28.45 & 0.164 \\
\hline 30.75 & 0.184 \\
\hline 33.06 & 0.209 \\
\hline 35.35 & 0.228 \\
\hline 37.72 & 0.247 \\
\hline 40.15 & 0.268 \\
\hline 42.60 & 0.289 \\
\hline 45.08 & 0.311 \\
\hline 47.58 & 0.332 \\
\hline 50.12 & 0.353 \\
\hline 52.96 & 0.375 \\
\hline 55.29 & 0.396 \\
\hline 57.92 & 0.417 \\
\hline 60.58 & 0.436 \\
\hline 63.26 & 0.456 \\
\hline
\end{tabular}


Table 2 (Continued)

\begin{tabular}{|c|c|}
\hline$T / K$ & $\frac{C_{p}}{J K^{-1} g^{-1}}$ \\
\hline 65.96 & 0.476 \\
\hline 68.69 & 0.495 \\
\hline 71.43 & 0.514 \\
\hline 74.19 & 0.532 \\
\hline 76.96 & 0.551 \\
\hline 79.75 & 0.568 \\
\hline 82.55 & 0.586 \\
\hline 85.36 & 0.603 \\
\hline 88.18 & 0.620 \\
\hline 91.01 & 0.637 \\
\hline 93.85 & 0.653 \\
\hline 96.71 & 0.669 \\
\hline 99.57 & 0.685 \\
\hline \multicolumn{2}{|l|}{ Series 7} \\
\hline 98.10 & 0.690 \\
\hline 98.20 & 0.675 \\
\hline 99.75 & 0.689 \\
\hline 102.69 & 0.709 \\
\hline 105.57 & 0.724 \\
\hline 108.45 & 0.737 \\
\hline 111.34 & 0.755 \\
\hline 114.23 & 0.771 \\
\hline 117.13 & 0.787 \\
\hline 120.03 & 0.802 \\
\hline 122.94 & 0.818 \\
\hline 125.85 & 0.833 \\
\hline 128.75 & 0.848 \\
\hline 131.66 & 0.863 \\
\hline 134.58 & 0.879 \\
\hline 137.50 & 0.894 \\
\hline 140.41 & 0.910 \\
\hline 143.33 & 0.926 \\
\hline 146.25 & 0.942 \\
\hline 149.17 & 0.957 \\
\hline 152.09 & 0.973 \\
\hline 155.01 & 0.989 \\
\hline 157.93 & 1.004 \\
\hline 160.85 & 1.022 \\
\hline 163.77 & 1.037 \\
\hline 166.69 & 1.053 \\
\hline 169.61 & 1.070 \\
\hline 172.52 & 1.085 \\
\hline 175.39 & 1.112 \\
\hline 178.26 & 1.099 \\
\hline 181.10 & 1.140 \\
\hline 183.88 & 1.155 \\
\hline 186.64 & 1.176 \\
\hline 189.37 & 1.198 \\
\hline 192.07 & 1.221 \\
\hline 194.75 & 1.242 \\
\hline 197.40 & 1.264 \\
\hline 200.03 & 1.286 \\
\hline 202.63 & 1.307 \\
\hline
\end{tabular}

Table 2 (Continued)

\begin{tabular}{|c|c|}
\hline$T / K$ & $\frac{C_{p}}{J K^{-1} g^{-1}}$ \\
\hline 205.20 & 1.328 \\
\hline 207.75 & 1.351 \\
\hline 210.27 & 1.375 \\
\hline 212.76 & 1.406 \\
\hline 215.21 & 1.444 \\
\hline 217.62 & 1.490 \\
\hline 219.99 & 1.542 \\
\hline 222.31 & 1.599 \\
\hline 224.58 & 1.656 \\
\hline 226.81 & 1.709 \\
\hline 229.00 & 1.758 \\
\hline 231.17 & 1.801 \\
\hline 233.30 & 1.839 \\
\hline 235.42 & 1.875 \\
\hline 237.51 & 1.908 \\
\hline 239.57 & 1.939 \\
\hline 241.62 & 1.968 \\
\hline 243.65 & 1.994 \\
\hline 245.66 & 2.021 \\
\hline 247.66 & 2.048 \\
\hline 249.63 & 2.077 \\
\hline 251.59 & 2.103 \\
\hline 253.54 & 2.128 \\
\hline 255.47 & 2.150 \\
\hline 257.39 & 2.175 \\
\hline 259.29 & 2.202 \\
\hline 261.19 & 2.228 \\
\hline 263.07 & 2.255 \\
\hline 264.94 & 2.282 \\
\hline 266.79 & 2.309 \\
\hline 268.63 & 2.336 \\
\hline 270.45 & 2.364 \\
\hline 272.26 & 2.390 \\
\hline 274.06 & 2.417 \\
\hline 275.84 & 2.445 \\
\hline 277.61 & 2.472 \\
\hline 279.37 & 2.499 \\
\hline \multicolumn{2}{|l|}{ Series 8} \\
\hline 280.24 & 2.437 \\
\hline 280.95 & 2.497 \\
\hline 282.26 & 2.551 \\
\hline 284.16 & 2.713 \\
\hline 286.01 & 2.803 \\
\hline 287.88 & 2.809 \\
\hline 289.78 & 2.795 \\
\hline 291.71 & 2.793 \\
\hline 293.65 & 2.803 \\
\hline 295.59 & 2.819 \\
\hline 297.53 & 2.840 \\
\hline 299.48 & 2.860 \\
\hline 301.44 & 2.881 \\
\hline 303.39 & 2.902 \\
\hline 305.35 & 2.924 \\
\hline
\end{tabular}


Table 2 (Continued)

\begin{tabular}{|c|c|}
\hline$T / K$ & $\frac{C_{p}}{J K^{-1} g^{-1}}$ \\
\hline 307.31 & 2.946 \\
\hline 309.27 & 2.971 \\
\hline 311.24 & 2.995 \\
\hline 313.20 & 3.023 \\
\hline 315.16 & 3.054 \\
\hline 317.13 & 3.087 \\
\hline 319.09 & 3.124 \\
\hline 321.06 & 3.163 \\
\hline 323.02 & 3.200 \\
\hline 324.99 & 3.236 \\
\hline 326.97 & 3.262 \\
\hline 328.95 & 3.281 \\
\hline 330.94 & 3.282 \\
\hline 332.92 & 3.275 \\
\hline 334.91 & 3.265 \\
\hline 336.91 & 3.253 \\
\hline 338.91 & 3.241 \\
\hline 340.91 & 3.221 \\
\hline 342.93 & 3.185 \\
\hline 344.96 & 3.119 \\
\hline 347.03 & 3.017 \\
\hline 349.15 & 2.883 \\
\hline 351.30 & 2.727 \\
\hline 353.47 & 2.576 \\
\hline 355.62 & 2.462 \\
\hline 357.73 & 2.410 \\
\hline 359.79 & 2.395 \\
\hline 361.81 & 2.395 \\
\hline 363.81 & 2.403 \\
\hline 365.80 & 2.408 \\
\hline 367.79 & 2.412 \\
\hline 369.78 & 2.417 \\
\hline 371.77 & 2.424 \\
\hline 373.76 & 2.432 \\
\hline 375.76 & 2.438 \\
\hline 377.75 & 2.442 \\
\hline 379.75 & 2.446 \\
\hline 381.75 & 2.448 \\
\hline 383.75 & 2.449 \\
\hline 385.75 & 2.456 \\
\hline 387.75 & 2.466 \\
\hline 389.74 & 2.470 \\
\hline 391.74 & 2.477 \\
\hline 393.74 & 2.485 \\
\hline 395.74 & 2.491 \\
\hline 397.75 & 2.496 \\
\hline 399.75 & 2.506 \\
\hline 401.75 & 2.512 \\
\hline 403.76 & 2.518 \\
\hline 405.77 & 2.524 \\
\hline 373.76 & 2.432 \\
\hline 375.76 & 2.438 \\
\hline
\end{tabular}

8 (up to about $300 \mathrm{~K}$ ) are also affected by relaxation because there was a waiting time of several hours under adiabatic conditions (almost constant temperature) between the end of series 7 and the start of series 8: first a lower heat capacity was observed than the heat capacities measured during series 3 and then the heat capacities were higher.

The results obtained in the temperature range from 350 to $406 \mathrm{~K}$ are plotted in Fig. 3. This temperature range represents the end of the melting process and the liquid state. Above $362 \mathrm{~K}$ the sample appears to be molten (series 3 and 8). During series 1, however, the end of melting was observed at a slightly higher temperature: $368 \mathrm{~K}$. Above $368 \mathrm{~K}$ the results of the series 1,3 and 8 are in almost perfect agreement with each other, although possibly in the temperature range 368-385 K the heat capacities measured during series 1 are slightly higher than those measured during series 3 and 8 . Above approximately $380 \mathrm{~K}$, within experimental error, the measurements are in very good agreement with (estimated) specific heat capacity data of (metastable) liquid LPE as given by Wunderlich and Czornyj [10] (and adopted by Mathot [11]):

$$
\begin{aligned}
\frac{c_{p}(\mathrm{LPE}, \mathrm{liq}, T)}{\mathrm{J} \mathrm{K}^{-1} \mathrm{~mol}^{-1}}= & 1.426+2.401 \times 10^{-3}\left(\frac{T}{\mathrm{~K}}\right) \\
& +7.065 \times 10^{-7}\left(\frac{T}{\mathrm{~K}}\right)^{2}
\end{aligned}
$$

Measurements on VLDPE showed a remarkable progressive increase of the specific heat capacity of the polymer in its liquid state between subsequent series of measurements [2]. This phenomenon was not observed for a homogeneous ethylene-1-octene copolymer [3] and for this EB 5 sample.

At the lowest temperatures, see Fig. $4 a-b$, the specific heat capacities are found to lie in between those for the reference states for completely amorphous and completely crystalline polyethylenes, as expected for a semi-crystalline polyethylene [11,12].

EB 5 shows a clear-cut glass transition, centred around $224 \mathrm{~K}$, which value marks the point of inflection of the heat capacity heating curve. The glass transition of ethylene-based copolymers is known to depend on the type and amount of comonomer [5] and the transition range for EB 5 is situated at lower temperatures as reported for BPE [13]. As typical for the present amount of comonomer, melting starts immediately on devitrification during heating. 


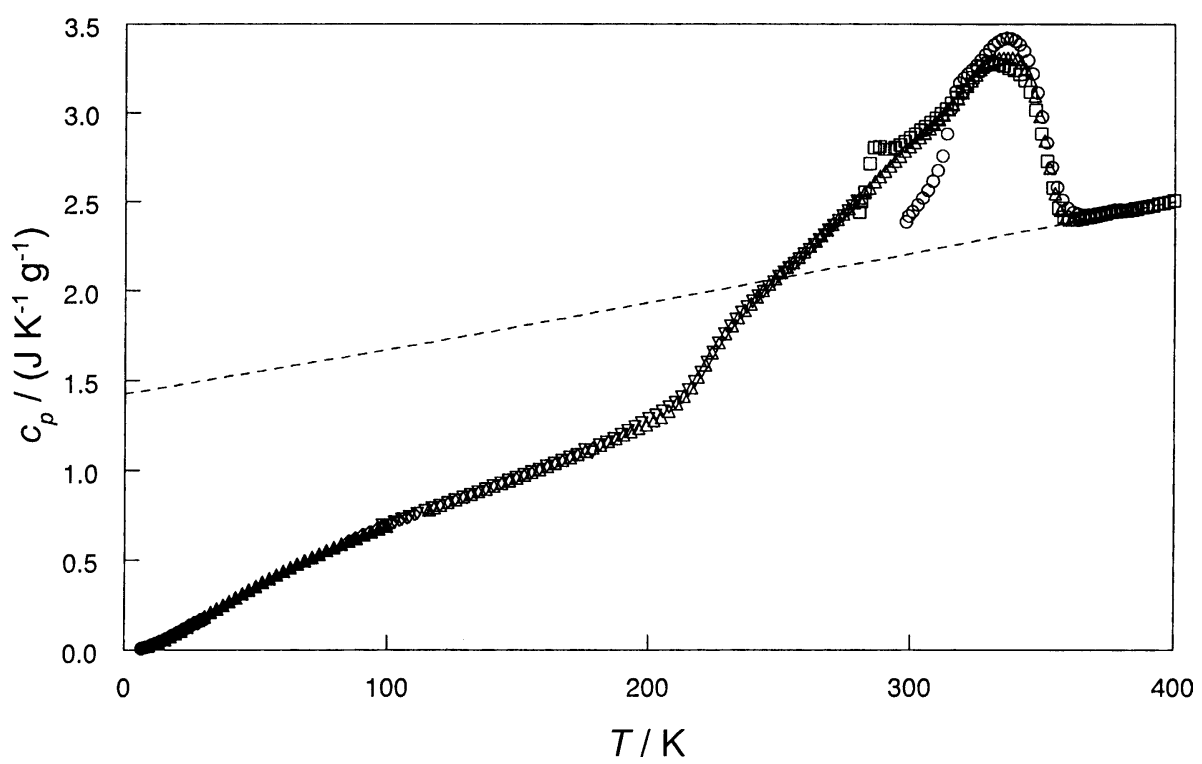

Fig. 2. Specific heat capacity of EB 5 as a function of temperature. Series 1 (sample as received) $(\bigcirc)$; series $2(\diamond)$; series $3(\triangle)$; series $4(\bullet)$; series $5(\diamond)$; series $6(\boldsymbol{\Delta})$; series $7(\nabla)$; series $8(\square)$. Dashed line, specific heat capacity of (metastable) liquid linear polyethylene (LPE) according to Eq. (1).

\subsection{Crystallinity}

The enthalpy-based mass fraction crystallinity of EB 5, as defined within the two-phase model, may be calculated using the following equation [12]:

$$
w^{\mathrm{c}}(T)=\frac{h_{\mathrm{a}}(T)-h(T)}{h_{\mathrm{a}}(T)-h_{\mathrm{c}}(T)}
$$

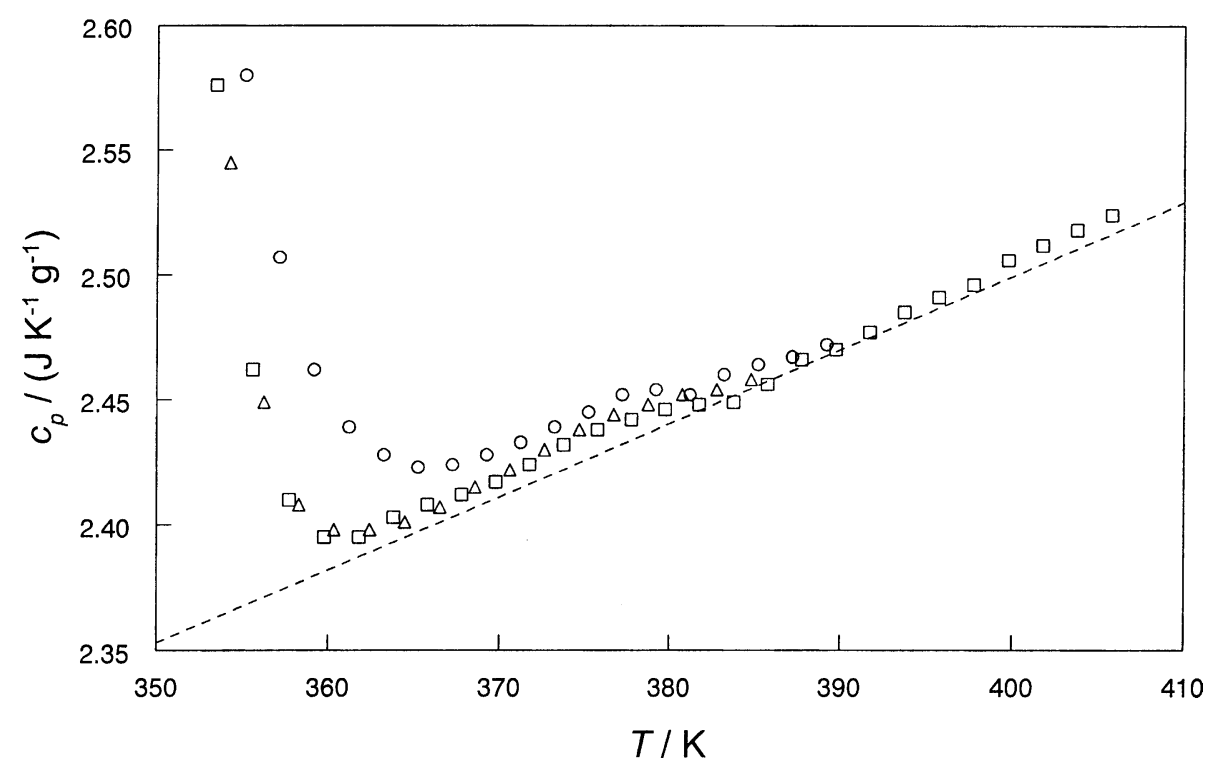

Fig. 3. Specific heat capacity of EB 5 in the liquid state. Series 1 (sample as received) $(\bigcirc)$; series 3 ( $\triangle$ ); series 8 ( $\square$ ). Dashed line, specific heat capacity of (metastable) liquid linear polyethylene (LPE) according to Eq. (1). 

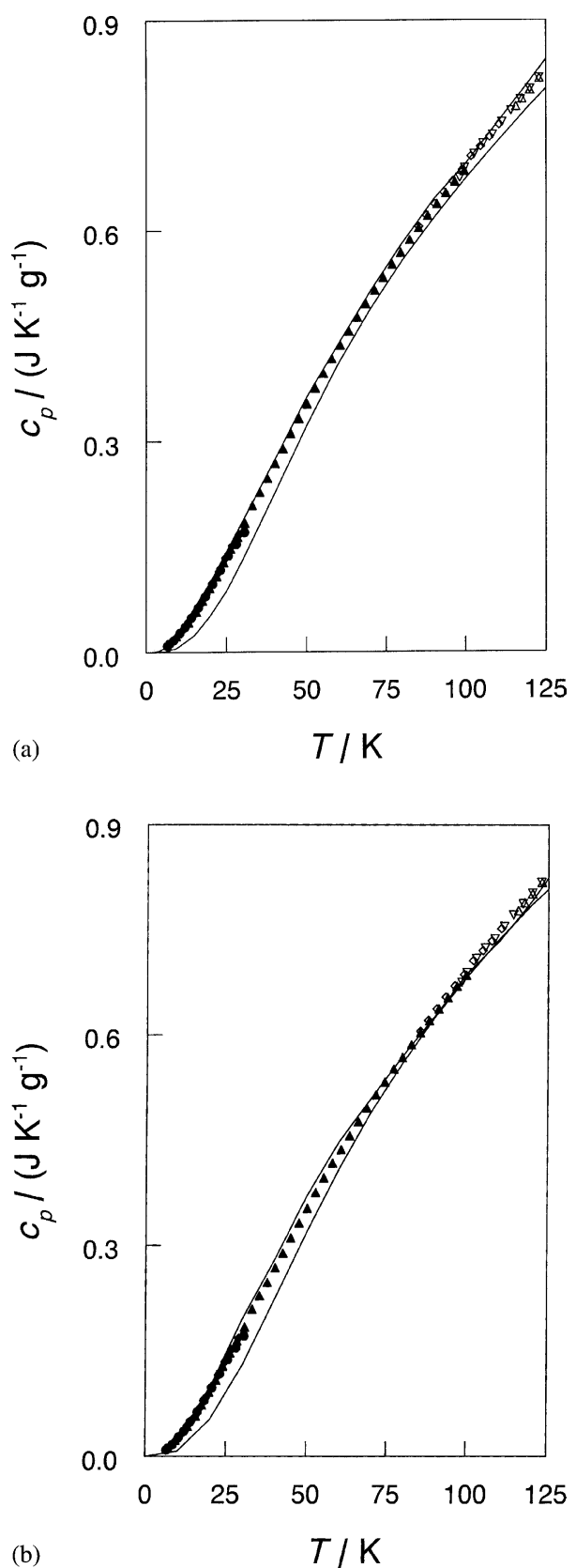

Fig. 4. Low temperature specific heat capacity data for EB 5. Series $2(\diamond)$; series $3(\triangle)$; series $4(\bullet)$; series $5(\bullet)$; series $6(\mathbf{\Delta})$; series $7(\nabla)$. (a) The solid lines represent specific heat capacity data for completely crystalline and completely amorphous polyethylene (BPE) according to the ATHAS data bank [13]; (b) the solid lines represent the data for completely amorphous and completely crystalline LPE according to Mathot [11]. Specific heat capacities for the completely amorphous phase are higher than those for the completely crystalline phase.
In this equation, $h_{\mathrm{a}}(T)$ is the specific enthalpy of the completely amorphous phase, $h_{\mathrm{c}}(T)$ the specific enthalpy of the completely crystalline phase and $h(T)$ the specific enthalpy of the semi-crystalline sample. Because the enthalpy function does not have a natural zero-point (see e.g. [14]) a common reference point has to be defined. Here, the specific enthalpy of EB 5 at a temperature of $400 \mathrm{~K}$ is selected as the reference point for the enthalpy function. At $T=400 \mathrm{~K} \mathrm{~EB} 5$ is completely molten; therefore, it may be stated that $h_{\mathrm{a}}(T=400 \mathrm{~K})=h(T=400 \mathrm{~K})$.

The specific enthalpy as a function of temperature is found by integrating the specific heat capacity:

$h(T=\Theta)-h(T=400 \mathrm{~K})=\int_{T=400 \mathrm{~K}}^{T=\Theta} c_{p}(T) \mathrm{d} T$

It is assumed here that the specific enthalpy of the crystalline phase is equal to the specific enthalpy of completely crystalline LPE as given by Mathot [11], corrected for the difference in reference point. The specific enthalpy of the amorphous phase is calculated using Eq. (3) by assuming that:

- the glass transition temperature is $T_{\mathrm{g}}=224 \mathrm{~K}$;

- the specific heat capacity of the amorphous phase above the glass transition temperature is equal to the specific heat capacity of (super-cooled) liquid LPE (given by Eq. (1));

- the specific heat capacity of the amorphous phase below the glass transition temperature is equal to the specific heat capacity of amorphous BPE in its glassy state as given by the ATHAS data bank [13].

The heat capacities used to evaluate the specific enthalpies are plotted in Fig. 5. The Fig. 6 represents a plot of the specific enthalpies thus found together with the enthalpy-based mass fraction crystallinity calculated using Eq. (2). In the temperature range from 220 to $360 \mathrm{~K}$ the crystallinity gradually decreases from 0.30 to zero, caused by melting of the crystalline phase. Vanden Eynde [4] calculated the crystallinity of EB 5 from DSC heating curves (after cooling at $10 \mathrm{~K} \mathrm{~min}^{-1}$ ): she found crystallinities of 0.26 at $233 \mathrm{~K}, 0.22$ at $273 \mathrm{~K}$ and 0.17 at $293 \mathrm{~K}$ whereas our values at the same temperatures are slightly higher: $0.28,0.24$ and 0.19 , respectively. This small difference is probably caused by the much smaller cooling rates in our experiments. 


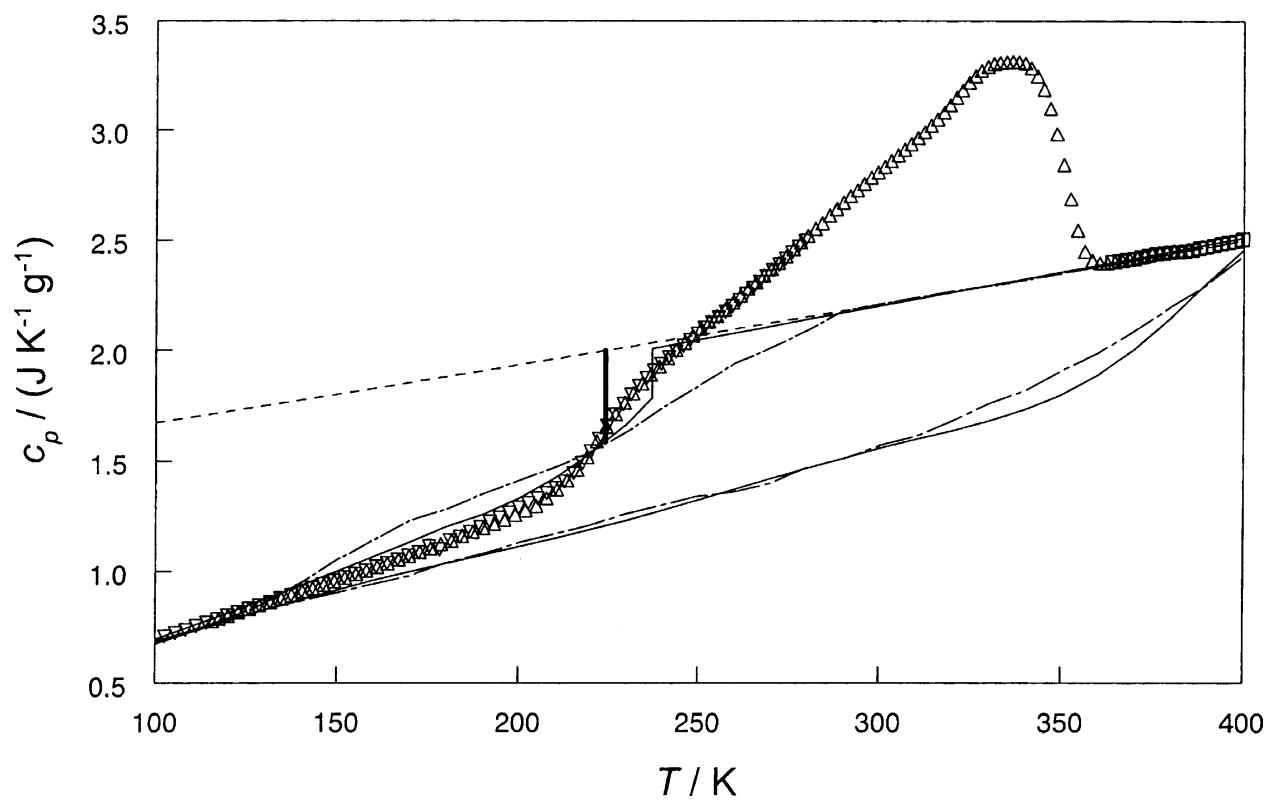

Fig. 5. Specific heat capacity data for EB 5 in the glass transition and melting region. Series $3(\triangle)$; series $7(\nabla)$; series $8(\square)$. The solid and the dash-dotted lines represent reference data for BPE according to the ATHAS data bank [13] and for LPE according to Mathot [11], respectively. Reference data are given for completely amorphous and for completely crystalline phases. The dashed line represents the specific heat capacity of (metastable) liquid LPE according to Eq. (1). The thick vertical line at $T=224 \mathrm{~K}$ represents the assumed heat capacity step for completely amorphous material at the glass transition temperature (see text).

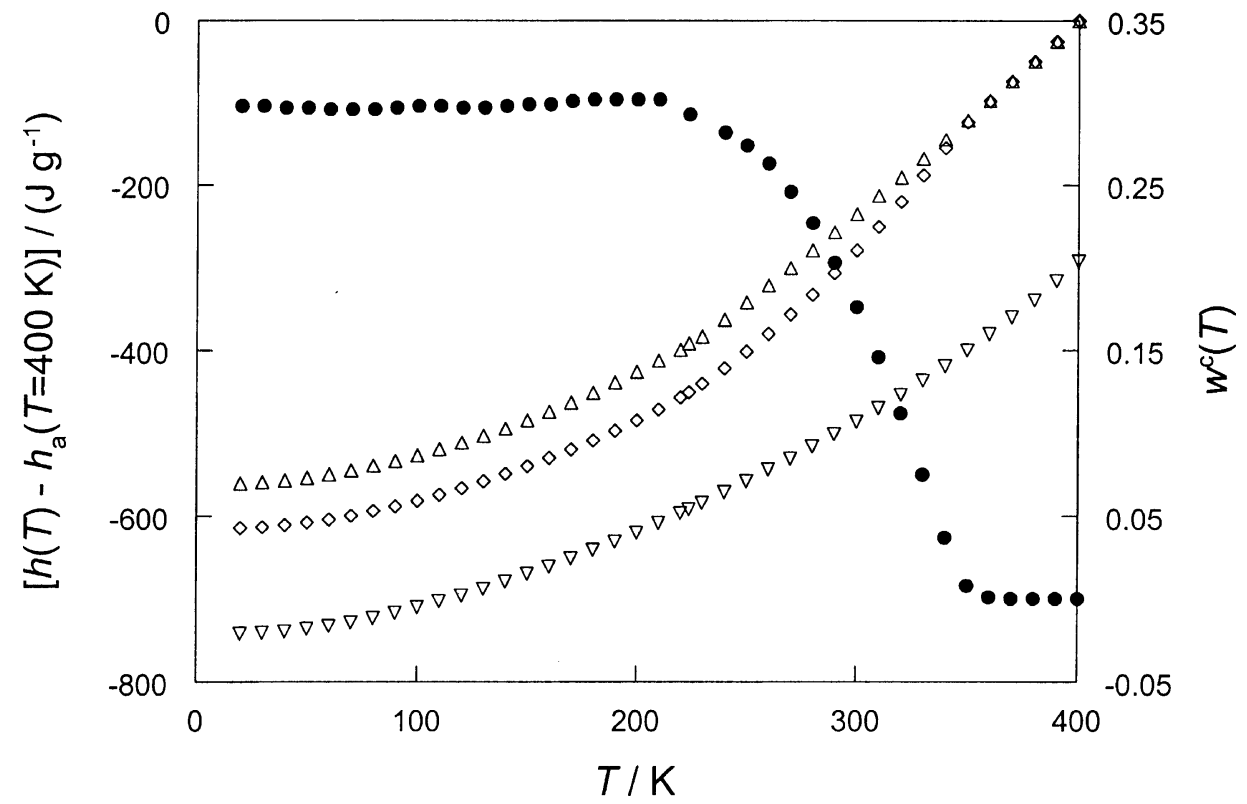

Fig. 6. Specific enthalpy of the semi-crystalline EB 5 sample $(\diamond)$, together with the specific enthalpy of the crystalline phase $(\nabla)$ and of the amorphous phase $(\triangle)$. The enthalpy-based mass fraction crystallinity (scaling given on the right vertical axis) of the EB 5 sample is also plotted (O). 


\subsection{Base line and excess heat capacities}

The so-called specific base-line heat capacity $\left(c_{p \mathrm{~b}}\right)$ [12] of EB 5 may be calculated using the evaluated crystallinity as a function of temperature and the following equation:

$c_{p \mathrm{~b}}(T)=w^{\mathrm{c}}(T) c_{p \mathrm{c}}(T)+\left[1-w^{\mathrm{c}}(T)\right] c_{p \mathrm{a}}(T)$

where $c_{p \mathrm{c}}$ and $c_{p \mathrm{a}}$ are the specific heat capacities of the completely crystalline and completely amorphous phases, respectively. Successively, the specific excess heat capacity $\left(c_{p e}\right)$ [12] can be calculated by subtracting the specific base-line heat capacity from the experimental specific heat capacity:

$c_{p \mathrm{e}}(T)=c_{p}(T)-c_{p \mathrm{~b}}(T)$

A plot of the specific base-line heat capacity and the specific excess heat capacity of EB 5 as a function of temperature is presented in Fig. 7.

\subsection{Temperature drifts during stabilisation periods in the melting region}

Temperature drifts of the sample and calorimeter vessel in the melting region were evaluated from linear fits of the temperature versus time curves in the second half of the stabilisation periods. The temperature drifts of measurement series $1,3,7$, and 8 are plotted in
Fig. 8. In a transition region characterised by an endothermic heat effect, such as melting, negative drifts are usually observed. The negative drifts for series 1 (up to $-180 \mu \mathrm{K} \mathrm{s}^{-1}$ ) and series 8 with local minima at approximately 315 and $285 \mathrm{~K}$, respectively, are related to the temperatures at which annealing took place (room temperature and $280 \mathrm{~K}$, respectively) and to the time it took (some years for series 1 and some hours for series 8). During series 1, 3, and 8 , however, at higher temperatures (above approximately $340 \mathrm{~K}$ for series 1 and above approximately $325 \mathrm{~K}$ for series 3 and 8 ) also positive temperature drifts were detected (see Fig. 8). This implies that in this part of the melting region a relaxation process occurs in the stabilisation periods, which must be intermitted as recrystallisation.

These observations for EB 5 are in agreement with the results that were obtained for a homogeneous ethylene-1-octene copolymer (EOM) [3] as well as for a heterogeneous ethylene-1-octene very low-density polyethylene [2].

Obviously, the longer the time spent in annealing, and/or the slower the cooling rate before subsequent melting, the more stable the material will be for an increasing temperature range above the annealing temperature. In these cases, during heating, possibilities of recrystallisation are decreased or even prohibited; in the latter case only melting is left.

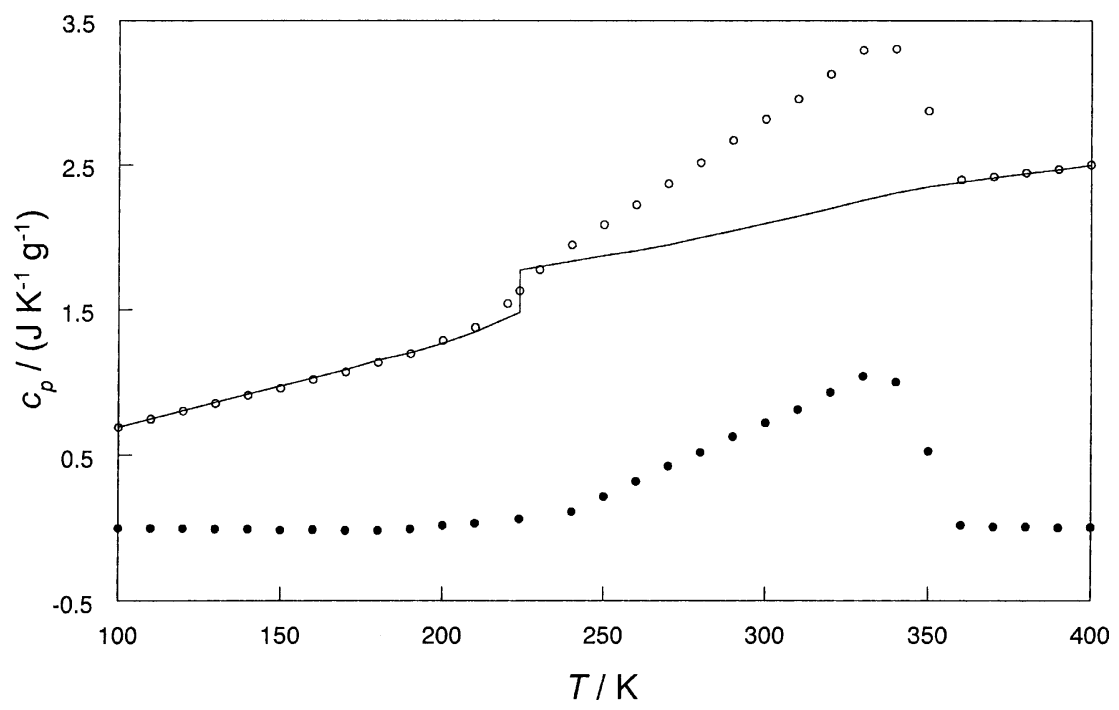

Fig. 7. Experimental specific heat capacity of EB $5(\bigcirc)$ together with the specific base-line heat capacity (solid line) and the specific excess heat capacity ( 


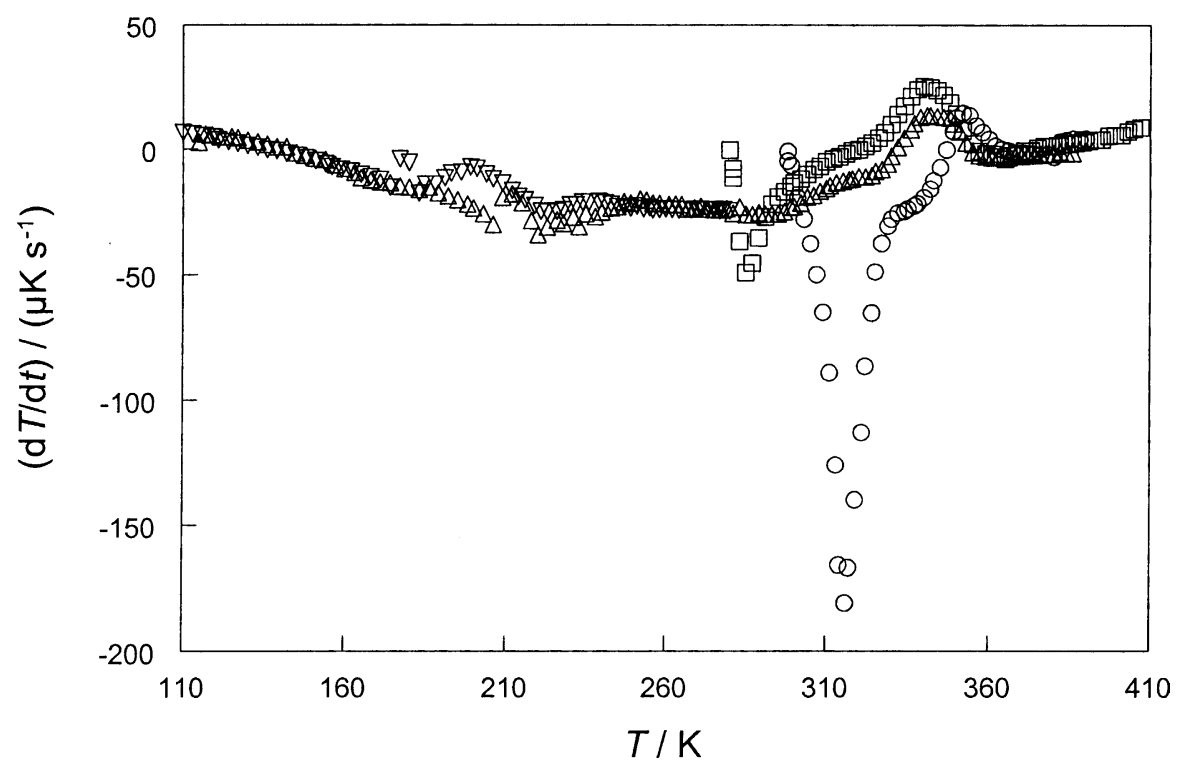

Fig. 8. Temperature drifts observed during the stabilisation periods. Series $1(\bigcirc)$ (sample as received); series $2(\triangle)$; series $3(\diamond)$; series $8(\square)$.

As stated in our paper presenting the results of measurements on a homogeneous ethylene-1-octene copolymer [3], the observed phenomena link up with temperature-modulated differential scanning calorimetry (TMDSC) experiments in which, depending on the thermal history, also excess phenomena are seen [15-17].

\section{References}

[1] V.B.F. Mathot (Ed.), Calorimetry and Thermal Analysis of Polymers, Cart Hanser, Munich, 1994.

[2] J.C. van Miltenburg, V.B.F. Mathot, P.J. van Ekeren, L.D. Ionescu, J. Therm. Anal. Cal. 56 (1999) 1017.

[3] P.J. van Ekeren, L.D. Ionescu, V.B.F. Mathot, J.C. van Miltenburg, J. Therm. Anal. Cal. 59 (2000) 683.

[4] S. Vanden Eynde, Homogeneous Ethylene-1-Alkene Copolymers: A Study of the Crystallisation and Melting Behaviour at Ambient and Elevated Pressures, Thesis Katholieke Universiteit Leuven, 1999.

[5] S. Vanden Eynde, V. Mathot, M.H.J. Koch, H. Reynaers, Polymer 42 (2000) 3437.
[6] J.C. van Miltenburg, G.J.K. van den Berg, M.J. van Bommel, J. Chem. Thermodyn. 19 (1987) 1129.

[7] J.C. van Miltenburg, A.C.G. van Genderen, G.J.K. van den Berg, Thermochim. Acta 319 (1998) 151.

[8] H. Preston-Thomas, Metrologia 27 (1990) 3.

[9] R.N. Goldberg, R.D. Weir, Pure Appl. Chem. 64 (1992) 1545.

[10] B. Wunderlich, G. Czornyj, Macromolecules 10 (1977) 906.

[11] V.B.F. Mathot, Polymer 25 (1984) 579.

[12] V.B. F. Mathot, Thermal Characterisation of States of Matter, in: V.B.F. Mathot (Ed.), Calorimetry and Thermal Analysis of Polymers, Cart Hanser, Munich, 1994, p. 105 (Chapter 5).

[13] ATHAS data bank, B. Wundelich, Pure Appl. Chem. 67 (1995) 1019. Detailed information may also be found on the internet: http://web.utk.edu/ athas.

[14] P.J. van Ekeren, Thermodynamic background to thermal analysis and calorimetry, in: M.E. Brown (Ed.), Handbook of Thermal Analysis and Calorimetry: Principles and Practice, Vol. 1, Elsevier, Amsterdam, 1998, p. 75 (Chapter 2).

[15] R. Scherrenberg, V. Mathot, A. van Hemelrijck, Thermochim. Acta 330 (1999) 3.

[16] R. Androsch, Polymer 40 (1999) 2805.

[17] A. Wurm, M. Merzlyakov, C. Schick, Thermochim. Acta 330 (1999) 121. 